\title{
Self-Organised Criticality as a Function of Connections' Number in the Model of the Rat Somatosensory Cortex
}

\author{
Grzegorz M. Wojcik and Wieslaw A. Kaminski \\ Institute of Computer Science \\ Maria Curie-Sklodowska University \\ pl. Marii Curie-Sklodowskiej 5, 20-031-Lublin, Poland \\ gmwojcik@gmail.com
}

\begin{abstract}
The model of the part of the rat somatosensory cortex was examined. Large network of Hodgkin-Huxley neurons was simulated and the modular architecture of this structure divided into layers and subregions was implemented. High degree of complexity required effective parallelisation of the simulation. In this article the results of the parallel neural computations are presented. An occurrence of the self-organised criticality was observed and its characteristics as a function of the connections number was investigated. It was proved that frequency of the socalled spike potential avalanches depends on the density of inter-neuron connections. In addition some benchmarking runs were conducted and parallelisation effectiveness is presented to some extent.
\end{abstract}

\section{Introduction}

The critical point is a point at which a system changes radically its behaviour or structure. Self-organised critical phenomena are defined by a complex system which reaches a critical state by its intrinsic dynamics, independently of a value of any control parameter. A typical example of a system exhibiting a self-organised criticality (SOC) is a sand pile model. The sand is slowly dropped onto a surface, forming a pile. As the pile grows, avalanches occur and they carry the sand from the top to the bottom of the pile. At least in the model, the slope of the pile becomes independent of the rate at which the system is driven by dropping sand. This exemplifies so-called (self-organised) critical slope [1].

The oldest numerical models describing the sand-pile problem are presented i.e., in [1], 3], 6]. In this model, a one-dimensional pile of sand is considered. Grains of sand are stored in the columns. The dynamics of the system is defined by a set of equations describing the effect of a one-grain addition. After a proper number of grains have been added to the appropriate columns, a critical inclination of the sand pile occurs and this causes disorder leading to relaxation of the whole system. This disorder is referred to as an avalanche.

Critical states of a system are signalled by a power-law distribution in some observable. In the case of sand-piles, the size and the distribution of the avalanches 
can be measured. A frequency of an avalanche occurrence in the system is a function of its size and can be expressed by the power law [1]:

$$
D(S) \sim S^{-k},
$$

where $k$ is always a characteristic number for a given system.

Complex systems exhibiting behaviour predicted by the SOC have been widely investigated [4], [5], 8], 10], 11], 14]. Earthquakes, forest fires, biological evolution are just three examples of wide range of phenomena that have been successfully modelled this way 1]. There are experiments that confirm the existence of frequency tuning and adaptation to stimulus statistics in neurons of the rat somatosensory cortex [7. SOC was found in the model of large biological neural networks [13, however, the aim of the research discussed in this contribution was to investigate whether and how the SOC occurrence depends on the number of connections in the simulated brain tissues. Good understanding the SOC mechanism in the model will allow us to design new series of experiments with the large number of interacting neurons leading to the discovery of new class of neurodynamical phenomena taking place in real brains.

Simulations of microcircuits consisting of numerically complicated HodgkinHuxley $(\mathrm{HH})$ neurons 9 are power consuming. The simulation time can be shortened by using cluster-based parallelised computing. All the simulations discussed in this paper were conducted in the parallel version of GENESIS compiled for the MPI environment [15. The choice of the GENESIS simulator allowed us to use many processors and to design effective way of parallelisation.

Remarkably, in this article we demonstrate that critical relaxation phenomena depend on the density of inter-neuron connections existing in the network. Consequently, the effectiveness of model's parallelisation, the simulation time and its speedup as a functions of the connections' number will be presented in the last section.

\section{Model and Method of Parallelisation}

The somatosensory pathways bring sensory information from the periphery into the brain, e.g., from a rat's whisker to the somatosensory cortex. Information from the snout passes along the trigeminal nerve, projecting to the trigeminal complex in the brainstem, which sends projections to the medial ventral posterior nucleus of the thalamus (VPm). Each whisker has a representative physical structure in the brain, forming 2-D maps of the whisker pad throughout the pathway. In the cortex, these structures are known as barrels. They are formed from clusters of stellate cortical neurons, with the cell bodies arranged in a ring and dendrites filling the middle "hole". The dendrites form synapses with multiple axons rising from the VPm [16].

The neurons chosen for the simulations were implemented according to the HH model 9]. Cells are relatively simple (for detail, see Appendix A). The only one modification in the model of neuron was arranged in order to avoid rapid synchronisation of the whole network: an additional parameter responsible 
for the probability of exocitosis was added for each synaptic connection in the post-synaptic neuron. Such a change required a simple modification of original GENESIS code. Changed version of GENESIS, compiled for Linux and MPI, can be downloaded from [15].

The simulated net consisted of 2025 of the above-mentioned neurons. All the cells were placed on a square-shaped grid with 45 rows and 45 columns. Each neuron was identified by a pair of numbers ranging from 0 to 44 . Network cells were divided into 22 groups, called layers, numbered from 1 to 22 . Communication between the neurons based on the following principles - the input signal from each cell from the $m$-th layer was transported to all the neurons from layers: $m+1, m+2, m+3, \ldots, m+N_{s}$, where $N_{s}$ was the integer number, not greater than the number of layers (see Fig. 1). Note that such a structure (2-D with dense "neural rings") imitates the structure of the rat's cortical barrels. The system can be easily parallelised, so we decided to simulate the problem on 15 processors. The network was divided into 15 zones. In each zone the same number of neurons was simulated. The zones were numbered from 1 to 15 and the way in which the zones were arranged is presented in Fig. 1. Such a choice allowed us to run simulations in optimal way, without the barriers being timed

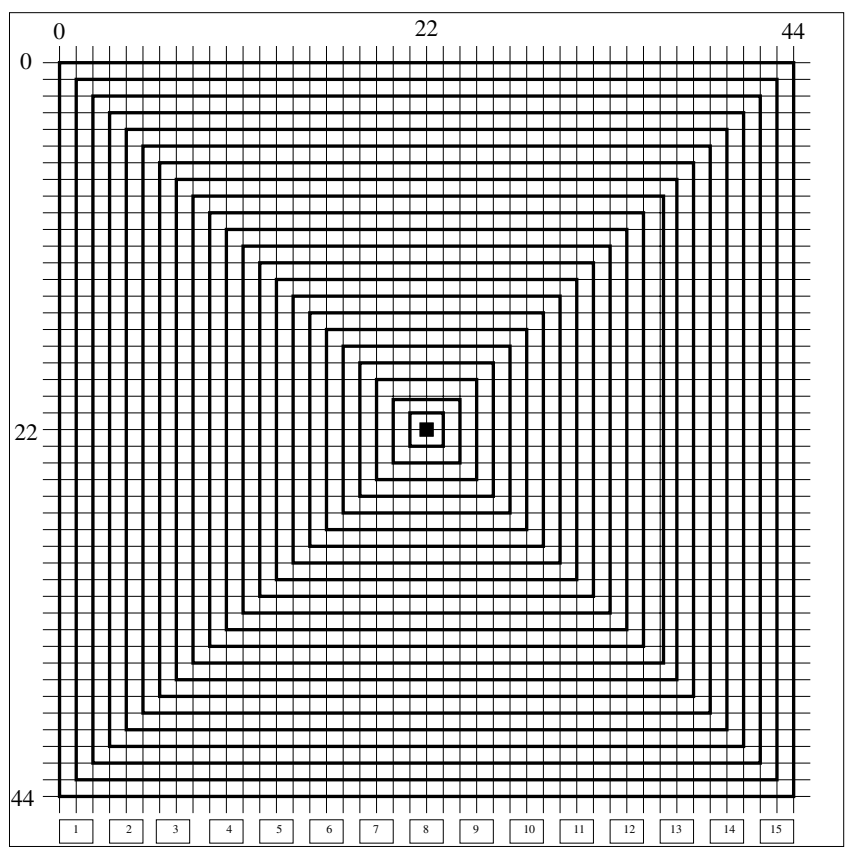

Fig. 1. Scheme of the simulated network. Layers are highlighted by thick lines. Stimulating neuron is marked with the black square and all other neurons are put on the intersections of grid lines. Neurons coordinates are marked on the top and the left side of the scheme. In each zone there are 3 columns of neurons as marked at the bottom. The choice of columns belonging to particular zones is arbitrary. 
out. The complexity of the system increases rapidly with $N_{s}$, so does the time of simulation. A good parallelisation of the model not only shortens its simulation time, but most often makes it executable at all. That is why parallelisation techniques are so important for HH systems with large number of synapses.

Synaptic connections were characterised by three parameters: weight $w$, time delay $\tau$, and the probability $p$ of transporting the signal, which corresponded to the mentioned probability for the occurrence of exocitosis. The probability $p$ was set to a constant, the same for all of the synapses $(p=0.5)$. Values of two other parameters depend on the position of both the pre-synaptic and postsynaptic neuron. For each pair of neurons from the $m$-th layer and the $n$-th layer respectively, the parameters $w$ and $\tau$ were chosen according to following rules:

$$
\begin{gathered}
w=\frac{w_{0}}{|m-n|}, \\
\tau=10^{-4}|m-n|[s],
\end{gathered}
$$

where $w_{0}$ was a positive constant (in our simulations $w_{0}=2$ ). The system was stimulated by the neuron $N[23,23]$ that was the main receptor of activities from the outside of the net (i.e., a glass capillary stimulating the whisker 7] or an electrode transmitting some random stimulus directly into the cortex). As a result, the receptor was producing a periodic spike potential with a frequency of about $80 \mathrm{~Hz}$. The net was characterised by the parameter $T$ that corresponded to the biological system's real working time (in these simulations $T=15 \mathrm{~s}$ ).

\section{Simulations and Results}

The stimulus was transported from the central unit to all other cells through the arranged connections. During the simulation, the time of spike potential occurrence was collected for each neuron. The avalanche occurs when the group of neurons is spiking in the same and small interval of time (i.e., $t_{i}=1 \mathrm{~ms}$ ). The algorithm used to compute the number of avalanches was implemented in $\mathrm{C}++$ (the simple analysis of text files containing the time and value of membrane potential, in search for the neurons having high spiking activity in the same time interval).

It was shown that for a system with a small number of neighbourhoods $\left(N_{s}<6\right)$ (the same small number of connections), the power law cannot precisely describe the number of spike-potential avalanche occurrences as a function of their size (Fig. 2). When the $N_{s}=6$ a kind of phase transition leading the system to the SOC behaviour can be observed (Fig. 3) 13. Systematic analysis of the SOC was performed i.e., by Peter Sloot 12 and the aim of our research described in this contribution was to investigate whether the occurrence of SOC depends only on the number of neighbourhoods or it can appear or disappear for a given $N_{s}$ as a function of the intra-network connections' number. As for the most sensitive parameter the value of 6 was chosen for the $N_{s}$ parameter in all series of the aforementioned experiments 13 . 


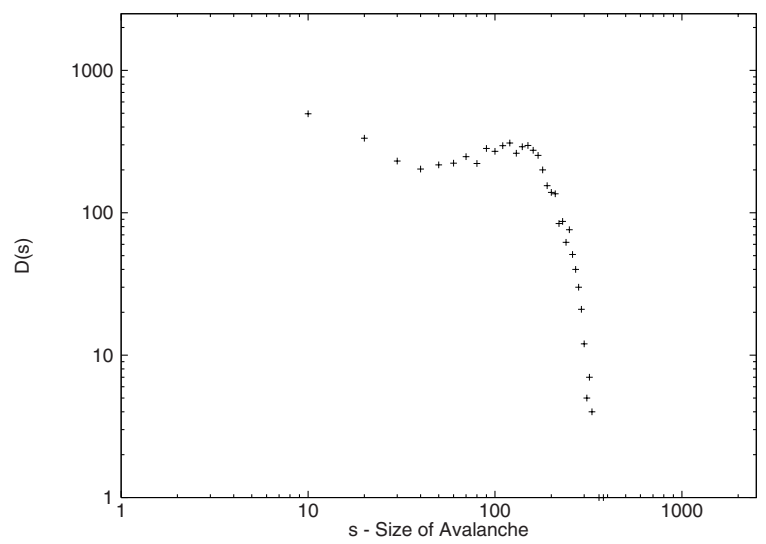

Fig. 2. Frequency $D(s)$ as the function avalanche size for $N_{s}=1, p_{x}=1, T=15 \mathrm{~s}$

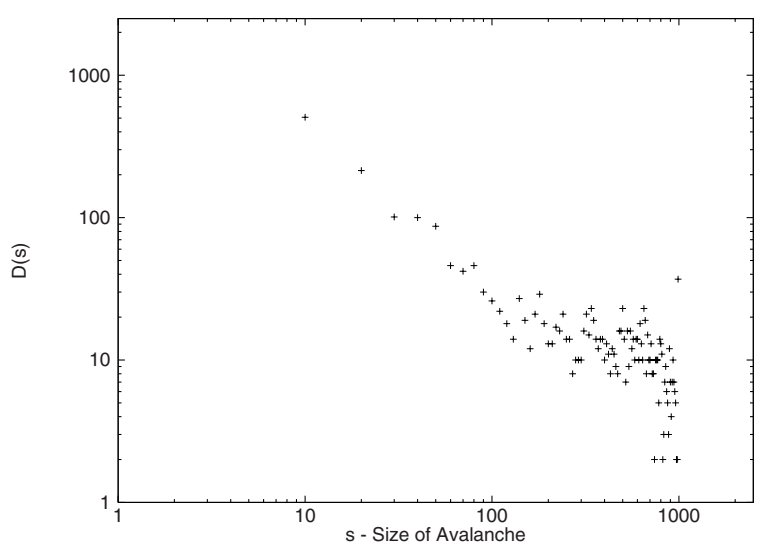

Fig. 3. Frequency $D(s)$ as the function of avalanche size for $N_{s}=6, p_{x}=1, T=15 \mathrm{~s}$

Then the another parameter $p_{x}$ defining the probability of synapse creation between two neurons from the the simulated network was implemented in the model. Surprisingly it was noted that self-organisation depends not only on the number of connections (what could be concluded from previous research) but also on the network architecture. On the basis of Fig. 2 and Fig. 3 one could hypothesise that when the number of connections falls the self-organisation disappears. However, for $N_{s}=6$ the SOC manifests itself even better when we decrease the strength of the inter-neuron communication by setting the $p_{x}$ below 0.07 (Fig. 4). What's more when $0.07<p_{x}<0.4$ the SOC behaviour tends to disappear (see two curves in Fig. 5) to come back for $p_{x}>0.4$ (Fig. 6). Because of relatively high system complexity and tendency to the rapid synchronisation the number of spikes decreases with the number of connections in the network (Fig. 7). That is why the number of avalanches and the inclination of the SOC 


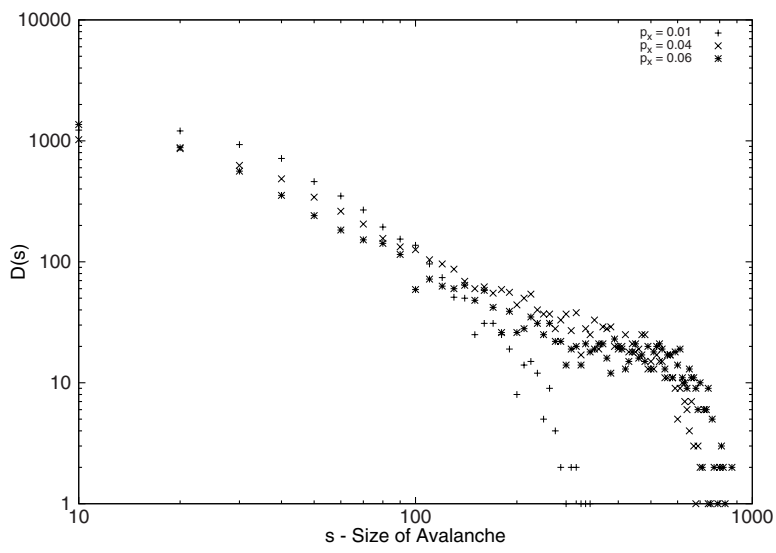

Fig. 4. Frequency $D(s)$ as a function of avalanche size for $N_{s}=6, p_{x}<0.07, T=15 \mathrm{~s}$

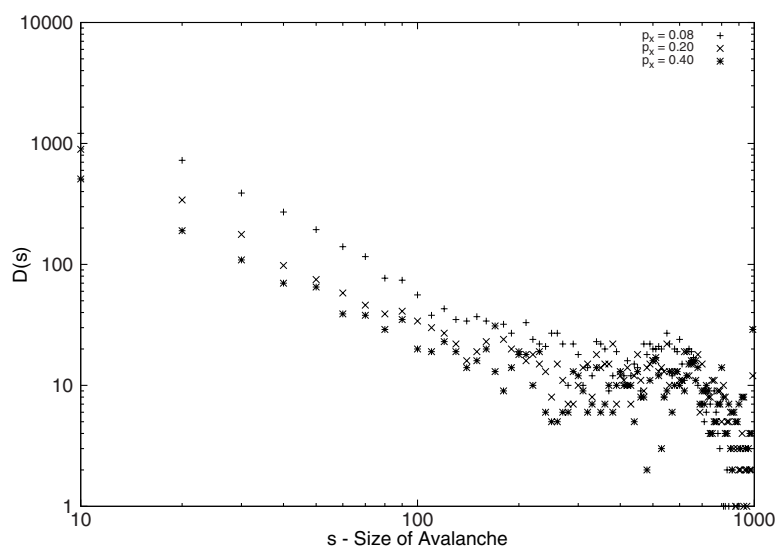

Fig. 5. Frequency $D(s)$ as a function avalanche size for $N_{s}=6,0.07<p_{x}<0.4$

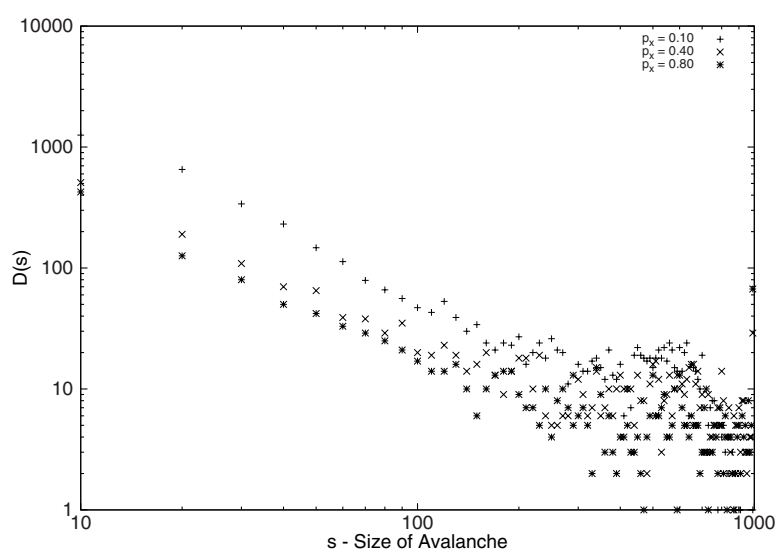

Fig. 6. Frequency $D(s)$ as a function avalanche size for $N_{s}=6, p_{x}>0.4, T=15 \mathrm{~s}$ 


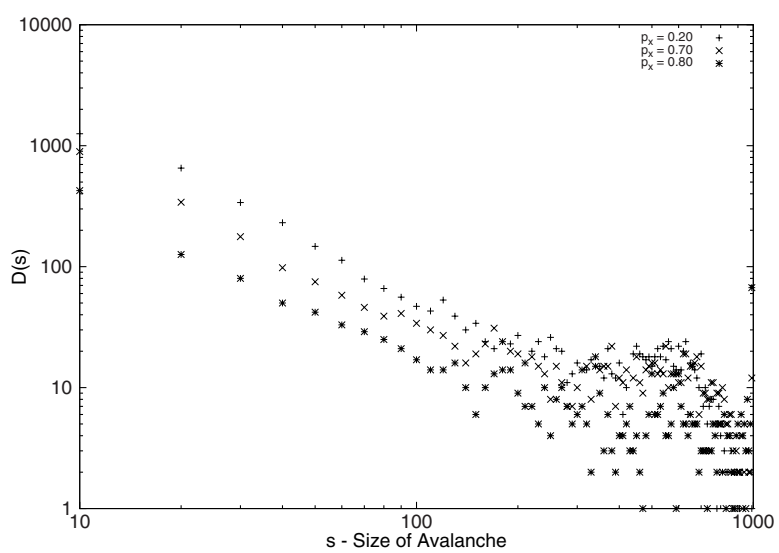

Fig. 7. Scale of the SOC for $N_{s}=6, T=15 \mathrm{~s}$. and different $p_{x}$

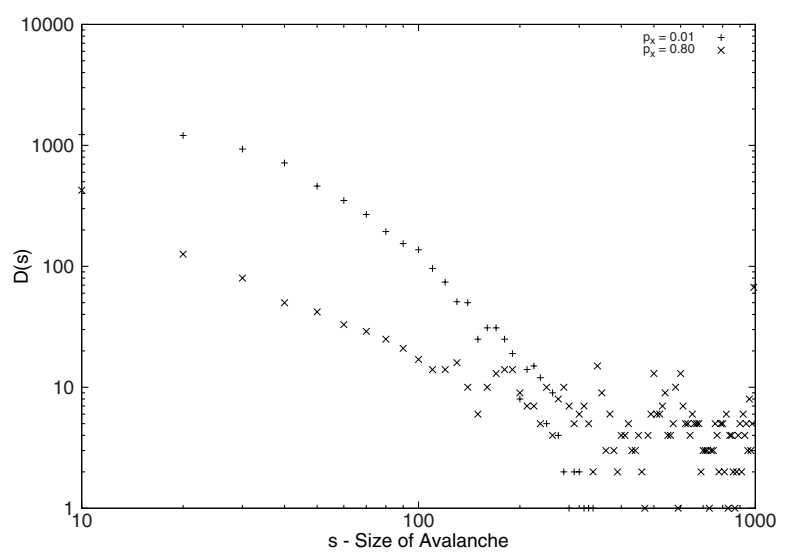

Fig. 8. SOC inclination for $N_{s}=6, T=15 \mathrm{~s}$. and different $p_{x}$

curve are different and depend on $p_{x}$ (Fig. 7-8). The number of spikes in the network falls with the growth of connections' density (Fig. 9).

\section{Parallelisation Effectiveness}

The local cluster used for all the simulations was built of 13 machines including one special machine - the so-called "access node". Each SMP machine had two 64-bit $1.4 \mathrm{GHz}$ Itanium2 IA64 processors with 4 GB of RAM memory. The cluster works under control of Debian Linux Sarge (v. 3.1) and 2.6.8-1 kernel version. The model is simulated in GEneral NEural Simulation System GENESIS v.2.2.1 with its MPI extension. A gcc compiler was used for the system compilation and in case of the MPI and Linux OS the compilation required some tuning of GENESIS code. Changed version can be found in [15]. 


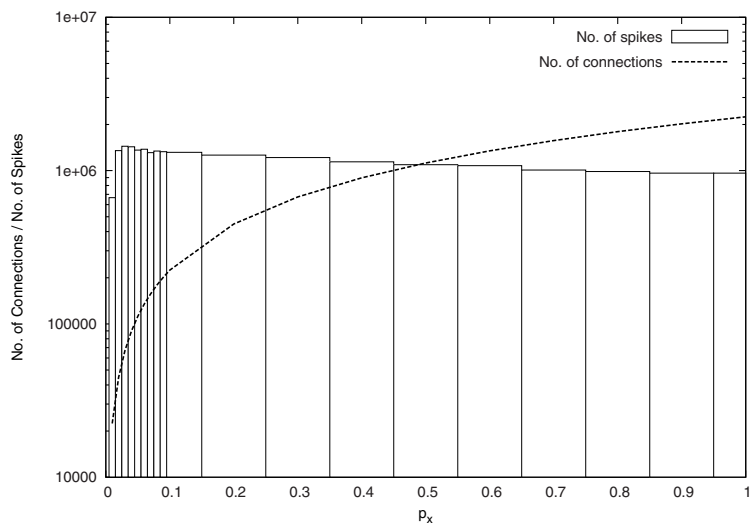

Fig. 9. Density of connections and number of spikes as a function of $p_{x}$

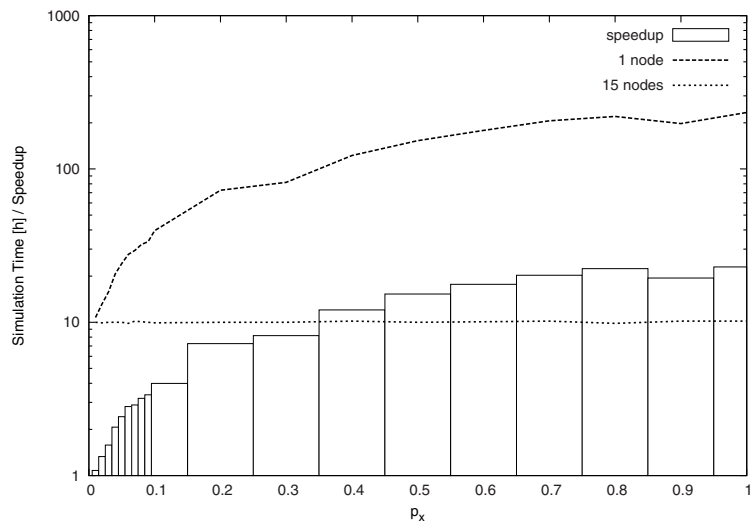

Fig. 10. Time of simulation and speedup as a function of $p_{x}$

The length of a typical run for $N_{s}=6$ and $T=15$ s was about 10 hours when the problem was parallelised for 15 nodes. However, for one node the simulation time ranged from $12 \mathrm{~h}$ to $230 \mathrm{~h}$ depending on the value of $p_{x}$. This gave us in the best case the speedup of 23 (Fig. 10). At first sight it is very optimistic result especially for the structures with large number of synapses. One should remember that 5 years ago such networks with $N_{s}>6$ modelled on one SPARC $400 \mathrm{MHz}$ node had the simulation time of about 3 weeks.

\section{Conclusions}

Systematic analysis of the simulated part of the rat's somatosensory cortex dynamics was conducted. Effective parallelisation was applied. SOC manifests itself in large biological neural networks. The "quality" of SOC depends both on the number of connections and on the architecture of the system. The role of SOC 
phenomena in mammalian brains is still unrecognised. However, good modelling will make it possible for us to design the new series of neuroscientific experiments, leading in the end to better understanding of the brain functionality.

\section{Appendix A: Properties of $\mathrm{HH}$ Neurons}

Our model consisted of multicompartmental neurons with two dendrite compartments, a soma and an axon. The dendrites contained a synaptically activated channel and the soma had voltage activated $\mathrm{HH}$ sodium and potassium channels. The behaviour of each compartment was equivalent to the behaviour of some electrical circuit 2. Thus, each circuit was characterised by a typical for GENESIS group of parameters set as follows: resistances $R_{a}=0.3 \Omega, R_{m}=0.33 \Omega$, capacity $C_{m}=0.01 \mathrm{~F}$, and potential $E_{m}=0.07 \mathrm{~V}$. For the soma compartment $E_{k}=0.0594 \mathrm{~V}$ and for the dendrite $E_{k}=0.07 \mathrm{~V}$. Conductance for each type of ionic channels was chosen to be: $G_{K}=360 \Omega^{-1}$ and $G_{N a}=1200 \Omega^{-1}$. These parameters originated from neurophysiological experiments [2] and were chosen to make the model biologically more realistic. The soma had a circular shape with the diameter of $30 \mu \mathrm{m}$, dendrites and axon were cable-like with the length of $100 \mu \mathrm{m}$. All the other parameters were chosen as suggested by GENESIS authors to simulate the behaviour of the biological-like neurons [2]. More details concerning the $\mathrm{HH}$ model can be found elsewhere [2], [9].

Acknowledgements. This work has been supported by the Maria Curie-Sklodowska University, Lublin, Poland (under the grant of UMCS Vice President 2007) and Polish State Committee for Scientific Research under the grant number (N519 017 32/2120). Special thanks to Peter Sloot for inspiration during the meeting in Russia.

\section{References}

1. Bak, P.: How nature works: The Science of Self-Organised Criticality. Copernicus Press, New York (1996)

2. Bower, J.M., Beeman, D.: The Book of GENESIS - Exploring Realistic Neural Models with the GEneral NEural SImulation System. Telos, New York (1995)

3. Jensen, H.J.: Self Organizing Criticality. Cambridge University Press, Cambridge (1998)

4. Aegerter, C.M., Gnther, R., Wijngaarden, R.J.: Avalanche dynamics, surface roughening, and self-organized criticality: Experiments on a three-dimensional pile of rice. Phys. Rev. E 67, 051306 (2003)

5. Bak, P., Christensen, K., Danon, L., Scanlon, T.: Unified Scaling Law for Earthquakes. Phys. Rev. Lett. 88, 178501 (2002)

6. Bak, P., Tang, C., Wisenfeld, K.: Self-organized criticality: An explanation of the 1/f noise. Phys. Rev. Lett. 59, 381-384 (1987)

7. Garcia-Lazaro, J.A., Ho, S.S.M., Nair, A., Schnupp, J.W.H.: Adaptation to Stimulus in Rat Somatosensory Cortex. FENS Abstr. 3, A109.4 (2006) 
8. Lubeck, S.: Crossover phenomenon in self-organized critical sandpile models. Phys. Rev. E 62, 6149-6154 (2000)

9. Hodgkin, A.L., Huxley, A.F.: A Quantitative Description of Membrane Current and its Application to Conduction and Excitation in nerve. J. Physiol. 117, 500-544 (1952)

10. Paczuski, M., Bassler, K.E.: Theoretical results for sandpile models of selforganized criticality with multiple topplings. Phys. Rev. E 62, 5347-5352 (2000)

11. Pastor-Satorras, R., Vespignani, A.: Corrections to scaling in the forest-fire model. Phys. Rev. E 61, 4854-4859 (2000)

12. Sloot, P.M.A., Overeinder, B.J., Schoneveld, A.: Self-organized criticality in simulated correlated systems. Comp. Phys. Comm. 142, 7-81 (2001)

13. Wojcik, G.M., Kaminski, W.A., Matejanka, P.: Self-organised Criticality in a Model of the Rat Somatosensory Cortex. In: Malyshkin, V.E. (ed.) PaCT 2007. LNCS, vol. 4671, pp. 468-476. Springer, Heidelberg (2007)

14. Yang, X., Du, S., Ma, J.: Do Earthquakes Exhibit Self-Organized Criticality? Phys. Rev. Lett. 92, 228501 (2004)

15. The GENESIS compiled for Linux MPI: http://www.luna.umcs.lublin.pl/download/modgenesis4mpi.tgz

16. The Rat Somatosensory Pathway: http://www.bris.ac.uk/Depts/Synaptic/info/pathway/somatosensory.htm 